\title{
Complex-dimensional Invariant Delta Functions and Lightcone Singularities
}

\author{
Noboru Nakanishi \\ Research Institute for Mathematical Sciences, Kyoto University, Kyoto 606, Japan
}

\begin{abstract}
Invariant delta functions (including imaginary-mass case) defined in a complex $n$-dimensional space-time are explicitly calculated in position space. It is proposed to define products of invariant delta functions in the ordinary Minkowski space by analytically continuing the corresponding $n$-dimensional ones to $n=4$. The (not only leading but also non-leading) lightcone singularities of $\left[\Delta\left(x ; m^{2}\right)\right]^{2}, \Delta\left(x ; m^{2}\right) \Delta^{(1)}\left(x ; m^{2}\right)$, and $\left[\Delta^{(1)}\left(x ; m^{2}\right)\right]^{2}$ are shown to be unambiguously determined in this way.
\end{abstract}

\section{Introduction}

Recently, much attention has been paid to the dimensional regularization method [1]. Though the Minkowski space is of course four-dimensional, Feynman integrals in momentum space can formally be extended to those in a complex $n$-dimensional space. Supposing that $\operatorname{Re} n$ is sufficiently small, one can calculate the latter without encountering ultraviolet divergences. Then one analytically continues the results to $n=4$ and thus obtains regularized Feynman integrals apart from possible poles located at $n=4$.

The purpose of the present paper is to apply this method to singular products in position space. Since invariant delta functions are singular on the lightcone, their naive products are meaningless as distributions. In order to give them reasonable definitions, we propose to use the dimensional regularization method.

In Section 2, we introduce the notion of complex $n$-dimensional Fourier transform and investigate its basic properties. In Section 3, we explicitly calculate the expressions for invariant delta functions in a complex $n$-dimensional spacetime. In Section 4 , the dimensional regularization method is applied to $\left[\Delta\left(x ; m^{2}\right)\right]^{2}$, $\Delta\left(x ; m^{2}\right) \Delta^{(1)}\left(x ; m^{2}\right)$, and $\left[\Delta^{(1)}\left(x ; m^{2}\right)\right]^{2}$, and the expressions for their lightcone singularities are explicitly found without encountering poles at $n=4$. Some discussions on our results are made in the final section.

In Appendix A, we present various formulae of Bessel functions which are used in this paper. Appendices B and C are devoted to the calculation needed in the text. 


\section{Complex-dimensional Fourier Transform}

We begin by defining a complex $n$-dimensional integral. It should be defined in such a way that it be analytic in $n$ and coincide with the ordinary $n$-ple integral when $n$ is a positive integer. Unfortunately, we cannot make use of Carlson's theorem to do this extension. Therefore we must introduce the complex $n$-dimensional integral in a direct way.

Let $p_{\mu}=\left(p_{0} ; p_{1}, \ldots, p_{k}, \ldots\right)$ be a complex $n$-dimensional Minkowski vector in an abstract sense. We define the complex $n$-dimensional integral of $F\left(p_{\mu}\right)$ by

$$
\begin{aligned}
\int d^{n} p F\left(p_{\mu}\right) \equiv & {\left[2 \pi^{(n-k-1) / 2} / \Gamma((n-k-1) / 2)\right] \int_{-\infty}^{\infty} d p_{0} \int_{-\infty}^{\infty} d p_{1} \ldots \int_{-\infty}^{\infty} d p_{k} } \\
& \cdot \int_{0}^{\infty} d p_{\perp} \cdot p_{\perp}{ }^{n-k-2} F\left(p_{0} ; p_{1}, \ldots, p_{k} ; p_{\perp}\right) .
\end{aligned}
$$

Here $F\left(p_{\mu}\right)$ is supposed to be a function of $k+1$ scalar products $x^{(1)} p, x^{(2)} p, \ldots, x^{(k)} p$ and $p^{2}$ together with $\varepsilon\left(p_{0}\right) \equiv p_{0} /\left|p_{0}\right|$. The spatial parts of $k$ complex $n$-dimensional fixed vectors $x^{(1)}{ }_{\mu}, \ldots, x^{(k)}{ }_{\mu}$ will generally span a $k$-dimensional subspace. The integration variables $p_{1}, \ldots, p_{k}$ are introduced as orthogonal coordinates in it, and $p_{\perp}$ is defined by

$$
p^{2} \equiv p_{0}{ }^{2}-p_{1}{ }^{2}-\ldots-{p_{k}}^{2}-{p_{\perp}}^{2} .
$$

If it happens that $F$ becomes independent of $p_{k}$, then, as is easily shown by setting $p_{\perp}^{\prime 2}=p_{\perp}^{2}+p_{k}^{2}$ and using a formula

$$
\int_{-1}^{1} d \xi\left(1-\xi^{2}\right)^{v}=\sqrt{\pi} \Gamma(v+1) / \Gamma(v+3 / 2),
$$

(2.1) reduces to

$$
\begin{aligned}
\int d^{n} p F\left(p_{\mu}\right)= & {\left[2 \pi^{(n-k) / 2} / \Gamma((n-k) / 2)\right] \int_{-\infty}^{\infty} d p_{0} \int_{-\infty}^{\infty} d p_{1} \ldots \int_{-\infty}^{\infty} d p_{k-1} } \\
& \cdot \int_{0}^{\infty} d p_{\perp}^{\prime} \cdot p_{\perp}^{\prime}{ }^{n-k-1} F\left(p_{0} ; p_{1}, \ldots, p_{k-1} ; p_{\perp}^{\prime}\right) .
\end{aligned}
$$

Thus our definition (2.1) does not intrinsically depend on $k$.

It is more convenient to introduce $k$ polar angles $\theta_{1}, \ldots, \theta_{k}$ and the radial length $|\boldsymbol{p}| \equiv\left(p_{1}{ }^{2}+\ldots+p_{k}{ }^{2}+p_{\perp}{ }^{2}\right)^{1 / 2}$. Then (2.1) is rewritten as

$$
\begin{aligned}
\int d^{n} p F\left(p_{\mu}\right)= & {\left[2 \pi^{(n-k-1) / 2} / \Gamma\left(\left(n-k^{-} 1\right) / 2\right)\right] \int_{-\infty}^{\infty} d p_{0} \int_{0}^{\infty} d|\boldsymbol{p}| \cdot|\boldsymbol{p}|^{n-2} \int_{0}^{\pi} d \theta_{1}\left(\sin \theta_{1}\right)^{n-3} } \\
& \ldots \int_{0}^{\pi} d \theta_{k}\left(\sin \theta_{k}\right)^{n-k-2} F\left(p_{0} ;|\boldsymbol{p}| ; \theta_{1}, \ldots, \theta_{k}\right) .
\end{aligned}
$$

The complex $n$-dimensional Fourier integral is a special case of (2.5), that is, the complex $n$-dimensional Fourier transform of $\varphi\left(p^{2}, \varepsilon\left(p_{0}\right)\right)$ is defined by

$$
\begin{aligned}
\int d^{n} p e^{-i p x} \varphi\left(p^{2}, \varepsilon\left(p_{0}\right)\right)= & {\left[2 \pi^{(n-2) / 2} / \Gamma((n-2) / 2)\right] \int_{-\infty}^{\infty} d p_{0} \int_{0}^{\infty} d|\boldsymbol{p}| \cdot|\boldsymbol{p}|^{n-2} } \\
& \cdot \int_{0}^{\pi} d \theta(\sin \theta)^{n-3} \exp \left[-i p_{0} x_{0}+i|\boldsymbol{p}| r \cos \theta\right] \varphi\left(p_{0}{ }^{2}-|\boldsymbol{p}|^{2}, \varepsilon\left(p_{0}\right)\right),
\end{aligned}
$$

where we formally set $x_{\mu}=\left(x_{0}, \boldsymbol{x}\right)$ and $|\boldsymbol{x}|=r$. 
Since our definition (2.1) is stable against the change of the number of scalar products involved in the integrand, we see that the abstract displacement of the integration vector is possible, that is,

$$
\int d^{n} p f\left(p x, p^{2}, \varepsilon\left(p_{0}\right)\right)=\int d^{n} p f\left((p-q) x,(p-q)^{2}, \varepsilon\left(p_{0}-q_{0}\right)\right) .
$$

From (2.7), we obtain the convolution formula

$$
\begin{aligned}
\int d^{n} p e^{-i p x} \int d^{n} q \varphi\left((p-q)^{2}, \varepsilon\left(p_{0}-q_{0}\right)\right) \psi\left(q^{2}, \varepsilon\left(q_{0}\right)\right)= & \int d^{n} p e^{-i p x} \varphi\left(p^{2}, \varepsilon\left(p_{0}\right)\right) \\
& \cdot \int d^{n} q e^{-i q x} \psi\left(q^{2}, \varepsilon\left(q_{0}\right)\right)
\end{aligned}
$$

for the complex $n$-dimensional Fourier transform.

Now, we prove Lorentz invariance of $(2.6)$. Since $\left[\varepsilon\left(p_{0}\right)\right]^{2}=1$, without loss of generality we may set

$$
\varphi\left(p^{2}, \varepsilon\left(p_{0}\right)\right)=\varphi\left(p^{2}\right)\left\{\begin{array}{c}
1 \\
i \varepsilon\left(p_{0}\right)
\end{array}\right\},
$$

where we of course assume that $\varphi\left(p^{2}\right)=0$ for $p^{2}<0$ in the lower row case.

The integration over $\theta$ is easily carried out by means of (A.18); then (2.6) reduces to

$$
\begin{aligned}
\int d^{n} p e^{-i p x} \varphi\left(p^{2}\right)\left\{\begin{array}{c}
1 \\
i \varepsilon\left(p_{0}\right)
\end{array}\right\}= & 2(2 \pi)^{(n-1) / 2} \int_{0}^{\infty} d p_{0} \int_{0}^{\infty} d|\boldsymbol{p}| \cdot|\boldsymbol{p}|^{n-2} \\
& \cdot \frac{J_{(n-3) / 2}(|\boldsymbol{p}| r)}{(|\boldsymbol{p}| r)^{(n-3) / 2}} \varphi\left(p_{0}{ }^{2}-|\boldsymbol{p}|^{2}\right)\left\{\begin{array}{c}
\cos p_{0} x_{0} \\
\sin p_{0} x_{0}
\end{array}\right\} \\
= & (2 \pi)^{(n-1) / 2} \int_{-\infty}^{\infty} d s \varphi(s) \chi_{n}\left(x_{0}, r ; s\right),
\end{aligned}
$$

where

$$
\chi_{n}\left(x_{0}, r ; s\right) \equiv \int_{0}^{\infty} d|\boldsymbol{p}| \frac{\theta\left(s+|\boldsymbol{p}|^{2}\right)}{\sqrt{s+|\boldsymbol{p}|^{2}}}|\boldsymbol{p}|^{n-2} \frac{J_{(n-3) / 2}(|\boldsymbol{p}| r)}{(|\boldsymbol{p}| r)^{(n-3) / 2}}\left\{\begin{array}{c}
\cos \sqrt{s+|\boldsymbol{p}|^{2}} x_{0} \\
\sin \sqrt{s+|\boldsymbol{p}|^{2}} x_{0}
\end{array}\right\} .
$$

For generic values of $r, x_{0}$, and $s$, this integral converges only if $1<\operatorname{Re} n<4$, but such restriction is unnecessary because of analytic continuation in $n$.

Lorentz invariance of (2.6) follows from that of (2.11). The latter is proved if $\chi_{n}\left(x_{0}, r ; s\right)$ is invariant under an infinitesimal transformation $\left(x_{0}, r\right) \rightarrow$ $\left(x_{0}+\varepsilon r, r+\varepsilon x_{0}\right)^{1}$.

To first order in $\varepsilon$, the increment of $\chi_{n}\left(x_{0}, r ; s\right)$ is

$$
\begin{aligned}
& \Delta \chi_{n}\left(x_{0}, r ; s\right)=\varepsilon \int_{0}^{\infty} d|\boldsymbol{p}| \frac{\theta\left(s+|\boldsymbol{p}|^{2}\right)}{\sqrt{s+|\boldsymbol{p}|^{2}}}|\boldsymbol{p}|^{n-2}\left[x_{0}\left(\frac{\partial}{\partial r} \frac{J_{(n-3) / 2}(|\boldsymbol{p}| r)}{(|\boldsymbol{p}| r)^{(n-3) / 2}}\right)\right. \\
& \left.\cdot\left\{\begin{array}{l}
\cos \sqrt{s+|\boldsymbol{p}|^{2}} x_{0} \\
\sin \sqrt{s+|\boldsymbol{p}|^{2}} x_{0}
\end{array}\right\}+\frac{J_{(n-3) / 2}(|\boldsymbol{p}| r)}{(|\boldsymbol{p}| r)^{(n-3) / 2}} r \frac{\partial}{\partial x_{0}}\left\{\begin{array}{l}
\cos \sqrt{s+|\boldsymbol{p}|^{2}} x_{0} \\
\sin \sqrt{s+|\boldsymbol{p}|^{2}} x_{0}
\end{array}\right\}\right] \text {. }
\end{aligned}
$$

\footnotetext{
1 Since (2.11) is non-singular at $r=0$ in general, we may assume $r>0$ so that $r+\varepsilon x_{0}>0$ for $\varepsilon$ infinitesimal
} 
The differentiation in the first term is easily carried out by means of (A.13). By using (A.14), we integrate the second term by parts:

$$
\begin{aligned}
& r \int_{0}^{\infty} d|\boldsymbol{p}|(|\boldsymbol{p}| r)^{(n-1) / 2} J_{(n-3) / 2}(|\boldsymbol{p}| r) \theta\left(s+|\boldsymbol{p}|^{2}\right)\left\{\begin{array}{r}
-\sin \sqrt{s+|\boldsymbol{p}|^{2}} x_{0} \\
\cos \sqrt{s+|\boldsymbol{p}|^{2}} x_{0}
\end{array}\right\} \\
& =-\int_{0}^{\infty} d|\boldsymbol{p}|(|\boldsymbol{p}| r)^{(n-1) / 2} J_{(n-1) / 2}(|\boldsymbol{p}| r)\left[\theta\left(s+|\boldsymbol{p}|^{2}\right)\left[\boldsymbol{p} \mid x_{0} / \sqrt{s+|\boldsymbol{p}|^{2}}\right]\right. \\
& \left.\cdot\left\{\begin{array}{l}
-\cos \sqrt{s+|\boldsymbol{p}|^{2}} x_{0} \\
-\sin \sqrt{s+|\boldsymbol{p}|^{2}} x_{0}
\end{array}\right\}+2|\boldsymbol{p}| \delta\left(s+|\boldsymbol{p}|^{2}\right)\left\{\begin{array}{r}
-\sin \sqrt{s+|\boldsymbol{p}|^{2}} x_{0} \\
\cos \sqrt{s+|\boldsymbol{p}|^{2}} x_{0}
\end{array}\right\}\right] .
\end{aligned}
$$

Then

$$
\begin{aligned}
\Delta \chi_{n}\left(x_{0}, r ; s\right)= & -\varepsilon r^{2-n} \int_{0}^{\infty} d|\boldsymbol{p}|(|\boldsymbol{p}| r)^{(n-1) / 2} J_{(n-1) / 2}(|\boldsymbol{p}| r) \\
& \cdot 2|\boldsymbol{p}| \delta\left(s+|\boldsymbol{p}|^{2}\right)\left\{\begin{array}{r}
-\sin \sqrt{s+|\boldsymbol{p}|^{2}} x_{0} \\
\cos \sqrt{s+|\boldsymbol{p}|^{2}} x_{0}
\end{array}\right\} .
\end{aligned}
$$

In the upper row case, (2.14) vanishes because $\delta\left(s+|\boldsymbol{p}|^{2}\right) \sin \sqrt{s+|\boldsymbol{p}|^{2}} x_{0}=0$, while in the lower row case it vanishes only if $s \geqq 0$ [but $\varphi(s)=0$ for $s<0$ by assumption]. Thus Lorentz invariance of (2.6) with (2.9) has been established.

Because of the even-oddness in $x_{0}$, (2.6) with (2.9) is a function of $x^{2}=x_{0}{ }^{2}-r^{2}$ only in the upper row case, while it is a function of $x^{2}$ multiplied by $\varepsilon\left(x_{0}\right)$ in the lower row case.

\section{Complex-dimensional Invariant Delta Functions}

The Fourier integral (2.6) is essentially a superposition of the following three invariant delta functions:

$$
\begin{aligned}
\Delta_{n}\left(x ; m^{2}\right) & \equiv\left(1 /(2 \pi)^{n-1} i\right) \int d^{n} p \varepsilon\left(p_{0}\right) \delta\left(p^{2}-m^{2}\right) e^{-i p x}, \\
\Delta^{(1)}\left(x ; m^{2}\right) & \equiv\left(1 /(2 \pi)^{n-1}\right) \int d^{n} p \delta\left(p^{2}-m^{2}\right) e^{-i p x}, \\
\Delta^{(2)}\left(x ; \mu^{2}\right) & \equiv\left(1 /(2 \pi)^{n-1}\right) \int d^{n} p \delta\left(p^{2}+\mu^{2}\right) e^{-i p x},
\end{aligned}
$$

where $m$ and $\mu$ are real constants.

We first calculate (3.1). From (2.10) with (2.11), we have

$$
\begin{aligned}
\Delta_{n}\left(x ; m^{2}\right)= & -\left[1 /(2 \pi)^{(n-1) / 2} r^{(n-3) / 2}\right] \int_{0}^{\infty} d|\boldsymbol{p}| \frac{|\boldsymbol{p}|^{(n-1) / 2}}{\sqrt{m^{2}+|\boldsymbol{p}|^{2}}} \\
& \cdot J_{(n-3) / 2}(|\boldsymbol{p}| r) \sin \sqrt{m^{2}+|\boldsymbol{p}|^{2}} x_{0} \\
= & -\left[1 /(2 \pi)^{(n-1) / 2} r^{(n-3) / 2}\right] \int_{m}^{\infty} d \omega\left(\omega^{2}-m^{2}\right)^{(n-3) / 4} \\
& \cdot J_{(n-3) / 2}\left(r \sqrt{\omega^{2}-m^{2}}\right) \sin \omega x_{0} .
\end{aligned}
$$

Hence (A.19) gives us

$$
\Delta_{n}\left(x ; m^{2}\right)=-\frac{\varepsilon\left(x_{0}\right) m^{(n-2) / 2}}{2^{n / 2} \pi^{(n-2) / 2}}\left(\sqrt{x^{2}}\right)^{(2-n) / 2} J_{(2-n) / 2}\left(m \sqrt{x^{2}}\right) \theta\left(x^{2}\right)
$$

for $1<\operatorname{Re} n<4$, but (3.5) can be analytically continued to any value of $n$. 
In order to see the lightcone singularities of (3.5), it is convenient to employ a distribution defined by

$$
\begin{aligned}
Y_{v}(v) & =\delta^{(k)}(v) \text { for } v=-k=0,-1,-2, \ldots, \\
& =\left(v^{v-1} / \Gamma(v)\right) \theta(v) \quad \text { otherwise. }
\end{aligned}
$$

It is well known in mathematics [2] that if $\varphi(v)$ is an arbitrary test function then the integral

$$
\int_{-0}^{\infty} d v \varphi(v) Y_{v}(v)
$$

is an entire function of $v$. Then using the series expansion (A.1) of $J_{v}$, we can rewrite (3.5) as

$$
\Delta_{n}\left(x ; m^{2}\right)=-\left(\varepsilon\left(x_{0}\right) / 2 \pi^{(n-2) / 2}\right) \sum_{l=0}^{\infty}\left((-1)^{l} / 2^{2 l} l !\right) m^{2 l} Y_{2-(n / 2)+l}\left(x^{2}\right) .
$$

It is easy to see that if $n=4$ (3.8) reproduces the well known expression for $\Delta\left(x ; m^{2}\right)$. In the form of (3.8), all lightcone singularities are manifest in any higher dimensional space-time.

For $m^{2}$ complex, (3.8) shows that $\Delta_{n}\left(x ; m^{2}\right)$ is an entire function of $m^{2}$. Thus (3.8) cán be used even for complex ghosts [3]. Furthermore, from (3.8) we have

$$
\left.\left(-\partial / \partial m^{2}\right)^{k} \Delta_{n}\left(x ; m^{2}\right)\right|_{m=0}=-\left(\varepsilon\left(x_{0}\right) / 2^{2 k+1} \pi^{(n-2) / 2}\right) Y_{2-(n / 2)+k}\left(x^{2}\right)
$$

for the massless multipole ghosts [3].

Next, we consider (3.2), which reduces to

$$
\begin{aligned}
\Delta_{n}^{(1)}\left(x ; m^{2}\right)= & \left(1 /(2 \pi)^{(n-1) / 2} r^{(n-3) / 2}\right) \int_{m}^{\infty} d \omega\left(\omega^{2}-m^{2}\right)^{(n-3) / 4} \\
& \cdot J_{(n-3) / 2}\left(r \sqrt{\omega^{2}-m^{2}}\right) \cos \omega x_{0} .
\end{aligned}
$$

Hence the use of (A.20) yields

$$
\begin{aligned}
\Delta_{n}^{(1)}\left(x ; m^{2}\right)= & \left(m^{(n-2) / 2} / 2^{n / 2} \pi^{(n-2) / 2}\right)\left[-\left(\sqrt{x^{2}}\right)^{(2-n) / 2} N_{(2-n) / 2}\left(m \sqrt{x^{2}}\right) \theta\left(x^{2}\right)\right. \\
& \left.+(2 / \pi)\left(\sqrt{-x^{2}}\right)^{(2-n) / 2} K_{(2-n) / 2}\left(m \sqrt{-x^{2}}\right) \theta\left(-x^{2}\right)\right]
\end{aligned}
$$

for $1<\operatorname{Re} n<4$, but, as before, (3.11) is valid for any value of $n$.

The modified Bessel function $K_{v}$ is usually expressed in terms of the Hankel function of the first kind $H^{(1)}{ }_{v}$ [see (A.4)]. In order to see the lightcone singularities of $\Delta^{(1)}{ }_{n}$ explicitly, it is more convenient to rewrite (3.11) as

$$
\begin{aligned}
\Delta_{n}^{(1)}\left(x ; m^{2}\right)= & \left(m^{n-2} / 2^{n / 2} \pi^{(n-2) / 2}\right) \cdot \frac{i}{2}\left[\left(m e^{i \pi / 2} \sqrt{-x^{2}+i 0}\right)^{(2-n) / 2}\right. \\
& \cdot H^{(1)}{ }_{(2-n) / 2}\left(m e^{i \pi / 2} \sqrt{-x^{2}+i 0}\right)+\left(m e^{i \pi / 2} \sqrt{-x^{2}-i 0}\right)^{(2-n) / 2} \\
& \cdot H^{(1)}{ }_{(2-n) / 2}\left(m e^{i \pi / 2} \sqrt{\left.-x^{2}-i 0\right)}\right] .
\end{aligned}
$$

This formula is verified in the following way. For $x^{2}<0,(3.12)$ directly reduces to the second term of (3.11). For $x^{2} \geqq 0$, since a branch cut is encountered, we should set $-x^{2}+i 0=e^{+i \pi} x^{2}$ and $-x^{2}-i 0=e^{-i \pi} x^{2}$. Then it is straightforward to show that (3.12) equals the first term of (3.11) by using (A.3), (A.7), and (A.2). 
A similar analysis for (3.5) yields

$$
\begin{aligned}
\Delta_{n}\left(x ; m^{2}\right)= & \frac{\varepsilon\left(x_{0}\right) m^{n-2}}{2^{n / 2} \pi^{(n-2) / 2}} \cdot \frac{1}{2}\left[\left(m e^{i \pi / 2} \sqrt{-x^{2}+i 0}\right)^{(2-n) / 2}\right. \\
& \cdot H_{(2-n) / 2}^{(1)}\left(m e^{i \pi / 2} \sqrt{-x^{2}+i 0}\right)-\left(m e^{i \pi / 2} \sqrt{-x^{2}-i 0}\right)^{(2-n) / 2} \\
& \cdot H_{(2-n) / 2}^{(1)}\left(m e^{i \pi / 2} \sqrt{\left.-x^{2}-i 0\right)}\right] .
\end{aligned}
$$

Accordingly, the complex $n$-dimensional Feynman propagator $\Delta_{F, n}\left(x ; m^{2}\right)$ is seen to be a boundary value of an analytic function:

$$
\begin{aligned}
\Delta_{F, n}\left(x ; m^{2}\right) \equiv & \frac{1}{2}\left[i \varepsilon\left(x_{0}\right) \Delta_{n}\left(x ; m^{2}\right)+\Delta_{n}^{(1)}\left(x ; m^{2}\right)\right] \\
= & \frac{m^{n-2} i}{2^{(n+2) / 2} \pi^{(n-2) / 2}}\left(m e^{i \pi / 2} \sqrt{\left.-x^{2}+i 0\right)^{(2-n) / 2}}\right. \\
& \cdot H_{(2-n) / 2}^{(1)}\left(m e^{i \pi / 2} \sqrt{-x^{2}+i 0}\right) .
\end{aligned}
$$

Finally, we discuss (3.3). Unfortunately, in this case, the author could not find an appropriate integration formula in the mathematical literature. Hence, on the basis of Lorentz invariance proved in Section 2, we calculate the "tachyon" [4] invariant delta function $\Delta^{(2)}{ }_{n}\left(x ; m^{2}\right)$ in two special cases $r=0$ and $x_{0}=0$. By means of (A.21) and (A.22), we respectively have

$$
\begin{aligned}
\left.\Delta_{n}^{(2)}\left(x ; \mu^{2}\right)\right|_{r=0} & =\frac{1}{(2 \pi)^{(n-1) / 2}} \cdot \frac{(1 / 2)^{(n-3) / 2}}{\Gamma((n-1) / 2)} \int_{0}^{\infty} d \omega\left(\omega^{2}+\mu^{2}\right)^{(n-3) / 2} \cos \omega x_{0} \\
& =\frac{\sin [(n-1) \pi / 2]}{2^{(n-2) / 2} \pi^{n / 2}}\left(\frac{x_{0}}{\mu}\right)^{(2-n) / 2} K_{(n-2) / 2}\left(\mu x_{0}\right), \\
\left.\Delta_{n}^{(2)}\left(x ; \mu^{2}\right)\right|_{x_{0}=0} & =\frac{1}{(2 \pi)^{(n-1) / 2} r^{(n-3) / 2}} \int_{\mu}^{\infty} d|\boldsymbol{p}| \frac{|\boldsymbol{p}|^{(n-1) / 2}}{\sqrt{|\boldsymbol{p}|^{2}-\mu^{2}}} J_{(n-3) / 2}(|\boldsymbol{p}| r) \\
& =-\frac{1}{2^{n / 2} \pi^{(n-2) / 2}}\left(\frac{r}{\mu}\right)^{(2-n) / 2} N_{(n-2) / 2}(\mu r)
\end{aligned}
$$

for $\operatorname{Re} n<4$. Thus

$$
\begin{aligned}
\Delta_{n}^{(2)}\left(x ; \mu^{2}\right)=- & \frac{\mu^{(n-2) / 2}}{2^{n / 2} \pi^{(n-2) / 2}}\left[\frac{2}{\pi} \cos \frac{n \pi}{2} \cdot\left(\sqrt{x^{2}}\right)^{(2-n) / 2}\right. \\
& \cdot K_{(n-2) / 2}\left(\mu \sqrt{x^{2}}\right) \theta\left(x^{2}\right)+\left(\sqrt{-x^{2}}\right)^{(2-n) / 2} \\
& \left.\cdot N_{(n-2) / 2}\left(\mu \sqrt{-x^{2}}\right) \theta\left(-x^{2}\right)\right]
\end{aligned}
$$

for any value of $n$. It is noteworthy that $\Delta^{(2)}{ }_{n}\left(x ; \mu^{2}\right)$ identically vanishes in the timelike region if and only if $n$ is an odd integer.

An analysis similar to the derivation of (3.12) shows that (3.17) can be rewritten as

$$
\begin{aligned}
\Delta_{n}^{(2)}\left(x ; \mu^{2}\right)=- & \frac{\mu^{n-2}}{2^{n / 2} \pi^{(n-2) / 2}} \cdot \frac{1}{2}\left[\left(\mu \sqrt{-x^{2}+i 0}\right)^{(2-n) / 2}\right. \\
& \cdot N_{(n-2) / 2}\left(\mu \sqrt{-x^{2}+i 0}\right)+\left(\mu \sqrt{-x^{2}-i 0}\right)^{(2-n) / 2} \\
& \cdot N_{(n-2) / 2}\left(\mu \sqrt{\left.-x^{2}-i 0\right)}\right] .
\end{aligned}
$$


Comparing (3.17) or (3.18) with the analytic continuation in $m$ of (3.11) or (3.12), we can prove the relation

$$
\frac{1}{2}\left[\Delta^{(1)}\left(x ; \mu^{2} e^{i \pi}\right)+\Delta^{(1)}\left(x ; \mu^{2} e^{-i \pi}\right)\right]=\Delta^{(2)}{ }_{n}\left(x ; \mu^{2}\right)
$$

with the aid of (A.2)-(A.4) together with (A.7).

The third invariant delta function [3] for an imaginary mass particle is defined and given by

$$
\begin{aligned}
\Delta_{n}^{(3)}\left(x ; \mu^{2}\right) & \equiv(1 / 2 i)\left[\Delta_{n}^{(1)}\left(x ; \mu^{2} e^{i \pi}\right)-\Delta_{n}^{(1)}\left(x ; \mu^{2} e^{-i \pi}\right)\right] \\
& =-\frac{\mu^{(n-2) / 2}}{2^{n / 2} \pi^{(n-2) / 2}}\left(\sqrt{-x^{2}}\right)^{(2-n) / 2} J_{(n-2) / 2}\left(\mu \sqrt{-x^{2}}\right) .
\end{aligned}
$$

Note that (3.20) is an entire function of $x^{2}$ for any value of $n$; therefore it has no lightcone singularity in any dimension.

\section{Lightcone Singularities of Products of Invariant Delta Functions}

As is well known and also as can be confirmed from (3.8) and (3.12), the invariant delta functions $\Delta\left(x ; m^{2}\right)$ and $\Delta^{(1)}\left(x ; m^{2}\right)$ in the ordinary Minkowski space have the following lightcone singularities:

$$
\begin{aligned}
\Delta\left(x ; m^{2}\right) & =-\frac{\varepsilon\left(x_{0}\right)}{2 \pi}\left[\delta\left(x^{2}\right)-\frac{m^{2}}{4} \theta\left(x^{2}\right)+\ldots\right], \\
\Delta^{(1)}\left(x ; m^{2}\right) & =-\frac{1}{2 \pi^{2}}\left[\mathrm{P} \frac{1}{x^{2}}-\frac{m^{2}}{4}\left(\log \frac{m^{2}\left|x^{2}\right|}{4}+2 \gamma-1\right)+\ldots\right],
\end{aligned}
$$

where P stands for Cauchy's principal value and $\gamma=0.5772 \ldots$ is Euler's constant. In the above, dots imply quantities of order $x^{2} \log \left|x^{2}\right|$ or higher.

As is evident from (4.1) and (4.2), such products as $\left[\Delta\left(x ; m^{2}\right)\right]^{2}$, $\Delta\left(x ; m^{2}\right) \Delta^{(1)}\left(x ; m^{2}\right)$ and $\left[\Delta^{(1)}\left(x ; m^{2}\right)\right]^{2}$ are mathematically meaningless in the sense of Schwartz's distribution [2]. One knows, however, that it is reasonable to set

$$
\delta(v) \mathrm{P} \frac{1}{v}=-\frac{1}{2} \delta^{\prime}(v) .
$$

Then the leading lightcone singularity of $\Delta\left(x ; m^{2}\right) \Delta^{(1)}\left(x ; m^{2}\right)$ is seen to be $-(2 \pi)^{-3} \varepsilon\left(x_{0}\right) \delta^{\prime}\left(x^{2}\right)^{2}$. But the non-leading lightcone singularity involves

$$
\mathrm{P} \frac{1}{x^{2}} \theta\left(x^{2}\right)+\delta\left(x^{2}\right) \log \left|x^{2}\right| \text {, }
$$

which remains undefined. In order to give natural definitions to those products of invariant delta functions, we propose to use the complex $n$-dimensional invariant delta functions, that is, we define the former by analytically continuing the corresponding products of the latter to $n=4$.

2 For a review on the lightcone singularities, see Frishman [5] 
First, we consider $\left[\Delta_{n}\left(x ; m^{2}\right)\right]^{2}$. From (3.5) and (A.23), we have

$$
\left[\Delta_{n}\left(x ; m^{2}\right)\right]^{2}=\frac{1}{4 \pi^{n-2}} \sum_{l=0}^{\infty} \frac{(-1)^{l} \Gamma(3-n+2 l)(m / 2)^{2 l}}{l ![\Gamma(2-(n / 2)+l)]^{2}} Y_{3-n+l}\left(x^{2}\right),
$$

where $Y_{v}$ is defined by (3.6). Hence its analytic continuation to $n=4$ is

$$
\lim _{n \rightarrow 4}\left[\Delta_{n}\left(x ; m^{2}\right)\right]^{2}=\frac{m^{2}}{16 \pi^{2}}\left[-\delta\left(x^{2}\right)+\frac{m^{2}}{4} \theta\left(x^{2}\right)+\ldots\right] .
$$

It is remarkable that the most singular term $(l=0)$ disappears. More generally, from (3.8) we can show that

$$
\lim _{n \rightarrow 4} \prod_{j=1}^{k} \Delta_{n}\left(x ; m_{j}^{2}\right)=\left[\frac{\varepsilon\left(x_{0}\right)}{8 \pi}\right]^{k}\left(\prod_{j=1}^{k} m_{j}^{2}\right)\left[-\frac{4}{k}\left(\sum_{j=1}^{k} \frac{1}{m_{j}^{2}}\right) \delta\left(x^{2}\right)+\theta\left(x^{2}\right)+\ldots\right] .
$$

Thus the lightcone singularities of products of $\Delta\left(x ; m_{j}^{2}\right)$ are essentially the same as those of $\Delta\left(x ; m^{2}\right)$. This result is characteristic to the even-dimensional space-time.

Next, we discuss $\Delta_{n}\left(x ; m^{2}\right) \Delta^{(1)}{ }_{n}\left(x ; m^{2}\right)$. In this case, direct computation of the lightcone singularities as above is impossible because of the presence of Neumann's Bessel function. By using the convolution theorem (2.8), it is straightforward to compute the Fourier transform of $\Delta_{n}\left(x ; m^{2}\right) \Delta^{(1)}{ }_{n}\left(x ; m^{2}\right)$ and to obtain

$$
\begin{aligned}
F_{n}(v)=- & \frac{(\sqrt{v})^{(2-n) / 2}}{2^{(5 n-6) / 2} \pi^{(2 n-3) / 2}} \int_{0}^{\infty} d s Y_{(n-1) / 2}\left(s-4 m^{2}\right)(\sqrt{s})^{(n-4) / 2} \\
& \cdot J_{(2-n) / 2}(\sqrt{s v)} \theta(v)
\end{aligned}
$$

with the aid of (3.5), where we have set

$$
F_{n}\left(x^{2}\right) \equiv \varepsilon\left(x_{0}\right) \Delta_{n}\left(x ; m^{2}\right) \Delta_{n}^{(1)}\left(x ; m^{2}\right) .
$$

But, unfortunately, (4.8) is not suitable for finding the lightcone singularities because even for the leading term the integration over $s$ is convergent only when $\operatorname{Re} n<2$.

A more convenient formula for our purpose is (A.24). On substituting (3.5) and (3.11) in (4.9) and using (A.24), we have

$$
F_{n}(v)=-\frac{1}{(2 \pi)^{n-1}}\left(\frac{\sqrt{v}}{m}\right)^{2-n} \int_{1}^{\infty} \frac{d u}{\sqrt{u^{2}-1}} J_{2-n}(2 m u \sqrt{v}) \theta(v) .
$$

Expanding $J_{2-n}$ into a power series, we obtain

$$
\begin{aligned}
F_{n}(v)=- & \frac{1}{(2 \pi)^{n-1}}\left[\sum_{l=0}^{k} \frac{(-1)^{l}}{l !} \cdot \frac{\sqrt{\pi} \Gamma((n-2 l-2) / 2)}{2 \Gamma((n-2 l-1) / 2)}\right. \\
& \left.\cdot m^{2 l} Y_{3-n+l}(v)+o\left(v^{3-n+k-\sigma}\right)\right], \quad(\sigma>0)
\end{aligned}
$$

where $k<(\operatorname{Re} n-2) / 2$ because of the convergence condition of the integration over $u$. For $n=4$, therefore, the leading lightcone singularity is found to be proportional to $\delta^{\prime}\left(x^{2}\right)$ in conformity with (4.3). 
It is a rather elaborative task to find the non-leading lightcone singularity; in working it out we have to keep in mind the fact that analytic continuation is possible only for exact expressions. As is expected from (4.4), we shall encounter an unfamiliar distribution; hence we should compute

$$
\int_{-0}^{\infty} d v \varphi(v) F_{n}(v)
$$

rather than (4.10) itself. For simplicity, we restrict test functions $\varphi(v)$ to functions holomorphic at $v=0$. Then in order to find the lightcone singularities, we have only to compute

$$
\Phi_{j}(\varepsilon) \equiv \lim _{n \rightarrow 4} \int_{-0}^{\varepsilon} d v \cdot v^{j} F_{n}(v), \quad(j=0,1,2, \ldots)
$$

where $\lim _{n \rightarrow 4}$ stands for the analytic continuation to $n=4$ and $\varepsilon$ is a small, but strictly positive $e^{3}$, number. After lengthy calculation, which is presented in Appendix B, we obtain

$$
\begin{aligned}
\Phi_{0}(\varepsilon)= & -\frac{m^{2}}{(2 \pi)^{3}} \cdot \frac{\pi}{2}\left[J_{0}(m \sqrt{\varepsilon}) N_{0}(m \sqrt{\varepsilon})+J_{1}(m \sqrt{\varepsilon}) N_{1}(m \sqrt{\varepsilon})\right], \\
\Phi_{1}(\varepsilon)= & -\frac{m^{2}}{(2 \pi)^{3}} \varepsilon\left\{-\frac{\pi}{2}\left[J_{0}(m \sqrt{\varepsilon}) N_{0}(m \sqrt{\varepsilon})+J_{1}(m \sqrt{\varepsilon}) N_{1}(m \sqrt{\varepsilon})\right]\right. \\
& \left.+\frac{\pi}{2 m \sqrt{\varepsilon}}\left[J_{0}(m \sqrt{\varepsilon}) N_{1}(m \sqrt{\varepsilon})+J_{1}(m \sqrt{\varepsilon}) N_{0}(m \sqrt{\varepsilon})\right]\right\}, \\
\Phi_{j}(\varepsilon)= & -\frac{m^{2}}{(2 \pi)^{3}} \varepsilon^{j} \cdot \frac{\pi}{2}\left[-\sum_{k=1}^{j} \frac{2 k(-1)^{k} j !(j-2) !}{(j+k) !(j-k) !} J_{k}(m \sqrt{\varepsilon}) N_{k}(m \sqrt{\varepsilon})\right. \\
& \left.-(j-1)^{-1} J_{1}(m \sqrt{\varepsilon}) N_{1}(m \sqrt{\varepsilon})\right] \text { for } j \geqq 2 .
\end{aligned}
$$

It is quite noteworthy that we encounter no higher transcendental functions other than Bessel functions.

As is shown in Appendix C, to order $\varepsilon^{j}$ (4.14), (4.15), and (4.16) become

$$
\begin{aligned}
& \Phi_{0}(\varepsilon) \sim-\frac{m^{2}}{(2 \pi)^{3}} \cdot \frac{1}{2}\left(\log \frac{m^{2} \varepsilon}{4}+2 \gamma-1\right), \\
& \Phi_{1}(\varepsilon) \sim-\frac{m^{2}}{(2 \pi)^{3}}\left(-\frac{1}{m^{2}}+\frac{\varepsilon}{2}\right), \\
& \Phi_{j}(\varepsilon) \sim-\frac{m^{2}}{(2 \pi)^{3}} \cdot \frac{\varepsilon^{j}}{2 j} \text { for } j \geqq 2,
\end{aligned}
$$

respectively.

3 This means that the order of $\varepsilon \rightarrow 0$ and $n \rightarrow 4$ cannot be interchanged 
Now, we consider a distribution $\operatorname{Pf}\left[v^{-1} \theta(v)\right]$, which is defined by

$$
\int_{-0}^{\varepsilon} d v \varphi(v) \operatorname{Pf}\left[v^{-1} \theta(v)\right] \equiv \varphi(\varepsilon) \log \varepsilon-\int_{0}^{\varepsilon} d v \varphi^{\prime}(v) \log v .
$$

This distribution was introduced previously by the present author [6] and by Wightman [7] independently. For $\varphi(v)=v^{j}$, we have

$$
\begin{aligned}
& \int_{-0}^{\varepsilon} d v \operatorname{Pf}\left[v^{-1} \theta(v)\right]=\log \varepsilon, \\
& \int_{-0}^{\varepsilon} d v \cdot v^{j} \operatorname{Pf}\left[v^{-1} \theta(v)\right]=\varepsilon^{j} / j \quad \text { for } \quad j \geqq 1 .
\end{aligned}
$$

Comparing (4.17)-(4.19) with (4.21) and (4.22), we find that the lightcone singularities of $\Delta\left(x ; m^{2}\right) \Delta^{(1)}\left(x ; m^{2}\right)$ are given by

$$
\begin{aligned}
\lim _{n \rightarrow 4} F_{n}\left(x^{2}\right)= & -\frac{1}{(2 \pi)^{3}}\left\{\delta^{\prime}\left(x^{2}\right)+\frac{m^{2}}{2} \operatorname{Pf}\left[\frac{1}{x^{2}} \theta\left(x^{2}\right)\right]\right. \\
& \left.+\frac{m^{2}}{2}\left(\log \frac{m^{2}}{4}+2 \gamma-1\right) \delta\left(x^{2}\right)+\ldots\right\} .
\end{aligned}
$$

The comparison of (4.23) with the naive expression for $\varepsilon\left(x_{0}\right) \Delta\left(x ; m^{2}\right) \Delta^{(1)}\left(x ; m^{2}\right)$ implies that the undefined expression (4.4) just corresponds to the distribution $\operatorname{Pf}\left[\left(1 / x^{2}\right) \theta\left(x^{2}\right)\right]^{4}$. In (4.23), the dots denote finite terms, which can be calculated from the naive expression if necessary.

Our final task is to investigate $\left[\Delta^{(1)}\left(x ; m^{2}\right)\right]^{2}$, but instead of dealing with it directly we consider

$$
G_{n}\left(x^{2}\right) \equiv\left[\Delta_{n}^{(1)}\left(x ; m^{2}\right)\right]^{2}-\left[\Delta_{n}\left(x ; m^{2}\right)\right]^{2} .
$$

On substituting (3.11) and (3.5) in (4.24) and using (A.25) and (A.26), we have

$$
\begin{aligned}
G_{n}(v) & =\frac{m^{n-2}}{2^{n-2} \pi^{n-1}}\left[-(\sqrt{v})^{2-n} \int_{1}^{\infty} \frac{d u}{\sqrt{u^{2}-1}} N_{2-n}(2 m u \sqrt{v}) \theta(v)\right. \\
& \left.+\frac{2}{\pi}(\sqrt{-v})^{2-n} \int_{1}^{\infty} \frac{d u}{\sqrt{u^{2}-1}} K_{2-n}(2 m u \sqrt{-v}) \theta(-v)\right] .
\end{aligned}
$$

The analysis of (4.24) is made quite analogously to that of $F_{n}\left(x^{2}\right)$, that is, as before, we can calculate

$$
\Psi_{j}(\varepsilon) \equiv \lim _{n \rightarrow 4} \int_{-\varepsilon}^{\varepsilon} d v \cdot v^{j} G_{n}(v) \quad(j=0,1,2, \ldots)
$$

\footnotetext{
4 This correspondence differs from the one given in Ref. [6] by a $\delta$-function multiplied by $\gamma$. Of course, the discrepancy is not serious because we are dealing with a mathematically meaningless expression. Indeed, the calculation (4.14) of Ref. [6] is ambiguous up to a constant multiple of a $\delta$-function
} 
exactly (see Appendix B). We thus obtain

$$
\begin{aligned}
\Psi_{0}(\varepsilon)= & \frac{m^{2}}{2^{4} \pi^{2}}\left\{\left[J_{0}(m \sqrt{\varepsilon})\right]^{2}-\left[N_{0}(m \sqrt{\varepsilon})\right]^{2}+\left[J_{1}(m \sqrt{\varepsilon})\right]^{2}\right. \\
& \left.-\left[N_{1}(m \sqrt{\varepsilon})\right]^{2}+\left[(2 / \pi) K_{0}(m \sqrt{\varepsilon})\right]^{2}-\left[(2 / \pi) K_{1}(m \sqrt{\varepsilon})\right]^{2}\right\}, \\
\Psi_{1}(\varepsilon)= & \frac{m^{2}}{2^{4} \pi^{2}} \varepsilon\left\{-\left[J_{0}(m \sqrt{\varepsilon})\right]^{2}+\left[N_{0}(m \sqrt{\varepsilon})\right]^{2}-\left[J_{1}(m \sqrt{\varepsilon})\right]^{2}\right. \\
& +\left[N_{1}(m \sqrt{\varepsilon})\right]^{2}+\left[(2 / \pi) K_{0}(m \sqrt{\varepsilon})\right]^{2}-\left[(2 / \pi) K_{1}(m \sqrt{\varepsilon})\right]^{2} \\
& +\frac{2}{m \sqrt{\varepsilon}}\left[J_{0}(m \sqrt{\varepsilon}) J_{1}(m \sqrt{\varepsilon})-N_{0}(m \sqrt{\varepsilon}) N_{1}(m \sqrt{\varepsilon})\right. \\
& \left.\left.+(2 / \pi)^{2} K_{0}(m \sqrt{\varepsilon}) K_{1}(m \sqrt{\varepsilon})\right]\right\}, \\
\Psi_{j}(\varepsilon)= & \frac{m^{2}}{2^{4} \pi^{2}} \varepsilon^{j}\left(\sum _ { k = 1 } ^ { j } \frac { 2 k \cdot j ! ( j - 2 ) ! } { ( j + k ) ! ( j - k ) ! } \left\{-(-1)^{k}\left[J_{k}(m \sqrt{\varepsilon})\right]^{2}\right.\right. \\
& \left.+(-1)^{k}\left[N_{k}(m \sqrt{\varepsilon})\right]^{2}-(-1)^{j}\left[(2 / \pi) K_{k}(m \sqrt{\varepsilon})\right]^{2}\right\} \\
& \left.+(j-1)^{-1}\left\{-\left[J_{1}(m \sqrt{\varepsilon})\right]^{2}+\left[N_{1}(m \sqrt{\varepsilon})\right]^{2}+(-1)^{j}\left[(2 / \pi) K_{1}(m \sqrt{\varepsilon})\right]^{2}\right\}\right) \\
& \text { for } j \geqq 2 .
\end{aligned}
$$

After somewhat complicated calculation, which is given in Appendix C, to order $\varepsilon^{j}$ we find

$$
\begin{aligned}
& \Psi_{0}(\varepsilon) \sim-\frac{1}{2 \pi^{4}} \cdot \frac{1}{\varepsilon}+\frac{m^{2}}{16 \pi^{2}}, \\
& \Psi_{j}(\varepsilon) \sim-\frac{m^{2}}{4 \pi^{4}} \cdot \frac{\varepsilon^{j}}{j}\left(\log \frac{m^{2} \varepsilon}{4}-\frac{1}{j}+2 \gamma-1\right) \text { for } j=1,3,5, \ldots, \\
& \Psi_{j}(\varepsilon) \sim \frac{1}{2 \pi^{4}} \cdot \frac{\varepsilon^{j-1}}{j-1} \text { for } j=2,4,6, \ldots
\end{aligned}
$$

Analogously to (4.20), we introduce a distribution $\operatorname{Pf}[(1 / v) \log |v|]$ by

$$
\int_{-\varepsilon}^{\varepsilon} d v \varphi(v) \operatorname{Pf}\left(\frac{1}{v} \log |v|\right) \equiv \frac{1}{2}[\varphi(\varepsilon)-\varphi(-\varepsilon)](\log \varepsilon)^{2}-\frac{1}{2} \int_{-\varepsilon}^{\varepsilon} d v \varphi^{\prime}(v)(\log |v|)^{2}
$$

so that

$$
\begin{aligned}
\int_{-\varepsilon}^{\varepsilon} d v \cdot v^{j} \operatorname{Pf}\left(\frac{1}{v} \log |v|\right) & =\frac{2 \varepsilon^{j}}{j}\left(\log \varepsilon-\frac{1}{j}\right) \text { for } j \text { odd } \\
& =0 \text { for } j \text { even }
\end{aligned}
$$

Hence the above results (4.30)-(4.32) can be expressed as

$$
\begin{aligned}
\lim _{n \rightarrow 4} G_{n}(v)= & \frac{1}{4 \pi^{4}}\left[\operatorname{Pf} \frac{1}{v^{2}}+\frac{\pi^{2}}{4} m^{2} \delta(v)-\frac{m^{2}}{2} \operatorname{Pf}\left(\frac{1}{v} \log |v|\right)\right. \\
& \left.-\frac{m^{2}}{4}\left(\log \frac{m^{2}}{4}+2 \gamma-1\right) \mathrm{P} \frac{1}{v}+\ldots\right],
\end{aligned}
$$


where $\operatorname{Pf} 1 / v^{2} \equiv-(d / d v)$ P $1 / v$ [2]. Comparing (4.35) with the naive expression for $\left[\Delta^{(1)}\left(x ; m^{2}\right)\right]^{2}-\left[\Delta\left(x ; m^{2}\right)\right]^{2}$, we see that $\operatorname{Pf} 1 / v^{2}$ and $\operatorname{Pf}[(1 / v) \log |v|]$ correspond to

$$
\left(\mathrm{P} \frac{1}{v}\right)^{2}-\pi^{2}[\delta(v)]^{2}
$$

and

$$
\log |v| \cdot \mathrm{P} \frac{1}{v}-\frac{\pi^{2}}{2} \varepsilon(v) \delta(v),
$$

respectively.

Finally, combining (4.35) with (4.6), we obtain

$$
\begin{aligned}
\lim _{n \rightarrow 4}\left[\Delta_{n}^{(1)}\left(x ; m^{2}\right)\right]^{2}= & \frac{1}{4 \pi^{4}}\left[\operatorname{Pf} \frac{1}{\left(x^{2}\right)^{2}}-\frac{m^{2}}{2} \operatorname{Pf}\left(\frac{1}{x^{2}} \log \left|x^{2}\right|\right)\right. \\
& \left.-\frac{m^{2}}{2}\left(\log \frac{m^{2}}{4}+2 \gamma-1\right) \mathrm{P} \frac{1}{x^{2}}+\ldots\right] .
\end{aligned}
$$

Note that (4.38) contains no $\delta$-type singularity.

\section{Discussions}

In the present paper, we have systematically investigated invariant delta functions in a complex $n$-dimensional space-time. As is shown in Section 3, the use of complex dimension allows us to discuss higher-dimensional invariant delta functions in a concise and unified way.

In Section 4, the complex $n$-dimensional method has been employed to regularize products of invariant delta functions in the ordinary Minkowski space. It is remarkable that we have encountered no poles at $n=4$, that is, no subtraction procedure has been necessary. Therefore our results are quite unambiguous.

Finally, we remark that $\Delta\left(x ; m^{2}\right) \Delta^{(1)}\left(x ; m^{2}\right)$ and $\left[\Delta^{(1)}\left(x ; m^{2}\right)\right]^{2}-\left[\Delta\left(x ; m^{2}\right)\right]^{2}$ can be defined by a different procedure. Since

$$
\begin{aligned}
2 \Delta_{F}\left(x ; m^{2}\right) & =i \varepsilon\left(x_{0}\right) \Delta\left(x ; m^{2}\right)+\Delta^{(1)}\left(x ; m^{2}\right) \\
& =-\frac{1}{2 \pi^{2}}\left[\frac{1}{x^{2}-i 0}-\frac{m^{2}}{4} \log \left(-x^{2}+i 0\right)-\frac{m^{2}}{4}\left(\log \frac{m^{2}}{4}+2 \gamma-1\right)+\ldots\right],
\end{aligned}
$$

we obtain

$$
\begin{aligned}
4\left[\Delta_{F}\left(x ; m^{2}\right)\right]^{2}= & {\left[\Delta^{(1)}\left(x ; m^{2}\right)\right]^{2}-\left[\Delta\left(x ; m^{2}\right)\right]^{2}+2 i \varepsilon\left(x_{0}\right) \Delta\left(x ; m^{2}\right) \Delta^{(1)}\left(x ; m^{2}\right) } \\
= & \frac{1}{4 \pi^{4}}\left[\frac{1}{\left(x^{2}-i 0\right)^{2}}-\frac{m^{2}}{2} \cdot \frac{1}{x^{2}-i 0} \log \left(-x^{2}+i 0\right)\right. \\
& \left.-\frac{m^{2}}{2}\left(\log \frac{m^{2}}{4}+2 \gamma-1\right) \frac{1}{x^{2}-i 0}+\ldots\right] .
\end{aligned}
$$


Since we know

$$
\begin{gathered}
\frac{1}{v-i 0}=\mathrm{P} \frac{1}{v}+i \pi \delta(v), \\
\frac{1}{(v-i 0)^{2}}=\mathrm{Pf} \frac{1}{v^{2}}-i \pi \delta^{\prime}(v)
\end{gathered}
$$

and can verify ${ }^{5}$

$$
\frac{1}{v-i 0} \log (-v+i 0)=\operatorname{Pf}\left(\frac{1}{v} \log |v|\right)-\frac{\pi^{2}}{2} \delta(v)+i \pi \operatorname{Pf}\left[\frac{1}{v} \theta(v)\right]
$$

(5.2) precisely reproduces the right-hand sides of (4.23) and (4.35).

Mathematically, we know that Schwartz's distribution can always be expressed as a linear combination of boundary values of analytic functions; the latter is called a "hyperfunction" by Sato [8]. Products of hyperfunctions are sometimes well defined on the basis of the defining analytic functions. As is seen above, $\Delta\left(x ; m^{2}\right) \Delta^{(1)}\left(x ; m^{2}\right)$ and $\left[\Delta^{(1)}\left(x ; m^{2}\right)\right]^{2}-\left[\Delta\left(x ; m^{2}\right)\right]^{2}$ are well defined, but $\left[\Delta\left(x ; m^{2}\right)\right]^{2}$ is still meaningless in the sense of hyperfunctions. Thus we may conclude that as far as invariant delta functions are concerned, our dimensional regularization method provides us a natural extension of the hyperfunction method in defining products of distributions.

\section{Appendix A. Bessel Function Formulae}

We here summarize Bessel function formulae which are applied in the present paper. We denote Bateman manuscript project's "Higher Transcendental Functions" Vol. 2 [9] by HTF and "Tables of Integral Transforms" Vols. 1 and 2 [10] by TIT $_{1}$ and by TIT $_{2}$, respectively.

The formulae (A.1)-(A.14) are found on pp. 4-12 (and on p. 79) of HTF. We denote Neumann's Bessel function by $N_{v}$ instead of $Y_{v}$ in order to distinguish it from the distribution defined in (3.6).

$$
\begin{aligned}
J_{v}(z) & =\sum_{l=0}^{\infty} \frac{(-1)^{l}(z / 2)^{v+2 l}}{l ! \Gamma(v+l+1)} \\
N_{v}(z) & =(\sin v \pi)^{-1}\left[J_{v}(z) \cos v \pi-J_{-v}(z)\right], \\
H^{(1)}(z) & =J_{v}(z)+i N_{v}(z)=(i \sin v \pi)^{-1}\left[J_{-v}(z)-J_{v}(z) e^{-i v \pi}\right], \\
K_{v}(z) & =(\pi / 2) i e^{i v \pi / 2} H_{v}^{(1)}\left(e^{i \pi / 2} z\right) .
\end{aligned}
$$

5 Integrate $\int_{-\varepsilon}^{\varepsilon} d v \varphi(v)(v-i 0)^{-1} \log (-v+i 0)$ by parts, use $\log (-v+i 0)=\log |v|+i \pi \theta(v)$ and compare the result with (4.20) and (4.33) 
For $k=0,1,2, \ldots$,

$$
\begin{aligned}
N_{k}(z)= & \frac{2}{\pi} J_{k}(z)\left(\gamma+\log \frac{z}{2}\right)-\frac{1}{\pi} \sum_{l=0}^{k-1} \frac{(k-l-1) !}{l !}\left(\frac{z}{2}\right)^{-k+2 l} \\
& -\frac{1}{\pi} \sum_{l=0}^{\infty} \frac{(-1)^{l}}{l !(k+l) !}\left(\frac{z}{2}\right)^{k+2 l}\left(h_{l}+h_{k+l}\right), \\
K_{k}(z)= & -(-1)^{k} \sum_{l=0}^{\infty} \frac{(z / 2)^{k+2 l}}{l !(k+l) !}\left(\gamma+\log \frac{z}{2}\right)+\frac{1}{2} \sum_{l=0}^{k-1} \frac{(-1)^{l}(k-l-1) !}{l !}\left(\frac{z}{2}\right)^{-k+2 l} \\
& +\frac{1}{2}(-1)^{k} \sum_{l=0}^{\infty} \frac{1}{l !(k+l) !}\left(\frac{z}{2}\right)^{k+2 l}\left(h_{l}+h_{k+l}\right),
\end{aligned}
$$

where $\gamma=0.5772 \ldots$ and $h_{l} \equiv \sum_{m=1}^{l} 1 / m$ with $h_{0}=0$.

$$
J_{v}\left(e^{i \pi} z\right)=e^{i v \pi} J_{v}(z)
$$

Let $Z_{v}$ be any one of $J_{v}, N_{v}$, and $H^{(1)}{ }_{v}$.

$$
\begin{aligned}
Z_{-k}(z) & =(-1)^{k} Z_{k}(z), \\
K_{-v}(z) & =K_{v}(z) ; \\
(2 v / z) Z_{v}(z) & =Z_{v-1}(z)+Z_{v+1}(z), \\
(2 v / z) K_{v}(z) & =-K_{v-1}(z)+K_{v+1}(z) ; \\
2(d / d z) Z_{v}(z) & =Z_{v-1}(z)-Z_{v+1}(z), \\
(d / d z)\left[z^{-v} Z_{v}(z)\right] & =-z^{-v} Z_{v+1}(z), \\
(d / d z)\left[z^{v+1} Z_{v+1}(z)\right] & =z^{v+1} Z_{v}(z) .
\end{aligned}
$$

Integrating both sides of (A.14) for $\operatorname{Re} v>-1$ and making a scale transformation, we have

$$
\begin{aligned}
& \int_{0}^{a} d z z^{v+1} J_{v}(y z)=a^{v+1} y^{-1} J_{v+1}(a y) \\
& \int_{0}^{a} d z z^{v+1} N_{v}(y z)=\frac{2^{v+1}}{\pi} \Gamma(v+1) y^{-v-2}+a^{v+1} y^{-1} N_{v+1}(a y)
\end{aligned}
$$

\section{Likewise}

$$
\int_{0}^{a} d z z^{v+1} K_{v}(y z)=2^{v} \Gamma(v+1) y^{-v-2}-a^{v+1} y^{-1} K_{v+1}(a y) .
$$


Formulae (A.18), (A.19), (A.20), and (A.21) are presented on p. 81 of HTF, on p. 113 of TIT $_{1}$, on p. 57 of TIT $_{1}$ [using (A.9) and correcting an obvious error] and on p. 11 of $\operatorname{TIT}_{1}$ [changing the sign of $v$ and using (A.9)], respectively.

$$
\begin{aligned}
& \int_{0}^{\pi} d \phi e^{i y \cos \phi}(\sin \phi)^{2 v}=2^{v} \sqrt{\pi} \Gamma(v+1 / 2) y^{-v} J_{v}(y) \quad \text { for } \operatorname{Re} v>-1 / 2 ; \\
& \int_{a}^{\infty} d z\left(z^{2}-a^{2}\right)^{v / 2} J_{v}\left(b \sqrt{z^{2}-a^{2}}\right) \sin y z \\
& =(\pi / 2)^{1 / 2} a^{(2 v+1) / 2} b^{v}\left(y^{2}-b^{2}\right)^{-(2 v+1) / 4} J_{-(2 v+1) / 2}\left(a \sqrt{y^{2}-b^{2}}\right) \theta(y-b) \\
& \quad \text { for } a>0, b>0, y>0,-1<\operatorname{Re} v<1 / 2, \\
& \int_{a}^{\infty} d z\left(z^{2}-a^{2}\right)^{v / 2} J_{v}\left(b \sqrt{z^{2}-a^{2}}\right) \cos y z \\
& =(2 / \pi)^{1 / 2} a^{(2 v+1) / 2} b^{v}\left(b^{2}-y^{2}\right)^{-(2 v+1) / 4} K_{-(2 v+1) / 2}\left(a \sqrt{b^{2}-y^{2}}\right) \theta(b-y) \\
& \quad-(\pi / 2)^{1 / 2} a^{(2 v+1) / 2} b^{v}\left(y^{2}-b^{2}\right)^{-(2 v+1) / 4} N_{-(2 v+1) / 2}\left(a \sqrt{y^{2}-b^{2}}\right) \theta(y-b) \\
& \text { for } a>0, b>0, y>0,-1<\operatorname{Re} v<1 / 2 ; \\
& \int_{0}^{\infty} d z\left(z^{2}+a^{2}\right)^{(2 v-1) / 2} \cos y z=\frac{2^{v} \sqrt{\pi}}{\Gamma(-v+1 / 2)} a^{v} y^{-v} K_{v}(a y) \\
& \text { for } y>0, \quad \operatorname{Re} v<1 / 2
\end{aligned}
$$

The following formula can be derived by combining the formula (32) on p. 25 of $\mathrm{TIT}_{2}$ (setting $\mu=-1 / 2$ and replacing $v$ by $-v$ ) and the formula (29) on p. 102 of $\mathrm{TIT}_{2}$.

$$
\begin{gathered}
\int_{a}^{\infty} d z z^{v+1}\left(z^{2}-a^{2}\right)^{-1 / 2} J_{v}(y z)=-(\pi / 2)^{1 / 2} a^{(2 v+1) / 2} y^{-1 / 2} N_{(2 v+1) / 2}(a y) \\
\text { for } y>0, a>0, \operatorname{Re} v<1 / 2 .
\end{gathered}
$$

The expansion formula

$$
J_{\mu}(z) J_{v}(z)=\sum_{l=0}^{\infty} \frac{(-1)^{l} \Gamma(\mu+v+2 l+1)(z / 2)^{\mu+v+2 l}}{l ! \Gamma(\mu+l+1) \Gamma(v+l+1) \Gamma(\mu+v+l+1)}
$$

is given on p. 11 of HTF, and the right-hand side is expressible in terms of a generalized hypergeometric function ${ }_{2} F_{3}$.

The formulae (A.24)-(A.26) are obtained from those presented on pp. 97, 96, and 54 of HTF by setting $\mu=v$ and $\cosh t=u$.

$$
\begin{aligned}
J_{v}(z) N_{v}(z) & =-\frac{2}{\pi} \int_{1}^{\infty} \frac{d u}{\sqrt{u^{2}-1}} J_{2 v}(2 z u), \\
{\left[J_{v}(z)\right]^{2}-\left[N_{v}(z)\right]^{2} } & =\frac{4}{\pi} \int_{1}^{\infty} \frac{d u}{\sqrt{u^{2}-1}} N_{2 v}(2 z u), \\
{\left[K_{v}(z)\right]^{2} } & =2 \int_{1}^{\infty} \frac{d u}{\sqrt{u^{2}-1}} K_{2 v}(2 z u) .
\end{aligned}
$$

They are valid for $z>0$. 


\section{Appendix B. Derivation of (4.14)-(4.16 and (4.27)-(4.29)}

The basic formula for the calculation of $\Phi_{j}(\varepsilon)$ is

$$
\int_{0}^{w} d v \cdot v^{v / 2} J_{v}(2 m u \sqrt{v})=\frac{w^{(v+1) / 2}}{m u} J_{v+1}(2 m u \sqrt{w}),
$$

which follows from (A.15).

We first calculate $\Phi_{0}(\varepsilon)$. The integration over $v$ is carried out by means of (B.1) with $w=\varepsilon$ and $v=2-n$. We analytically continue the result in $n$ and can set $n=4$ safely because $\varepsilon$ is non-zero. Hence

$$
\Phi_{0}(\varepsilon)=\frac{m^{2}}{(2 \pi)^{3}} \int_{1}^{\infty} \frac{d u}{\sqrt{u^{2}-1}} \cdot \frac{1}{m \sqrt{\varepsilon} u} J_{1}(2 m \sqrt{\varepsilon} u)
$$

with the aid of (A.8). We apply (A.10) to the integrand of (B.2). Then we can carry out the integration over $u$ by means of (A.24). Thus (4.14) is obtained.

Next, we consider $\Phi_{1}(\varepsilon)$. Integrating by parts with the aid of (B.1), we obtain

$$
\int_{0}^{\varepsilon} d v \cdot v \cdot(\sqrt{v})^{2-n} J_{2-n}(2 m u \sqrt{v})=\frac{\varepsilon^{(5-n) / 2}}{m u} J_{3-n}(2 m \sqrt{\varepsilon} u)-\frac{\varepsilon^{(4-n) / 2}}{(m u)^{2}} J_{4-n}(2 m \sqrt{\varepsilon} u) .
$$

We may set $n=4$ in (B.3) as before, but we do not do so because of a technical reason. Repeated application of (A.10) brings the right-hand side of (B.3) into

$$
\begin{aligned}
\frac{\varepsilon^{(6-n) / 2}}{5-n}\left\{J_{2-n}(2 m \sqrt{\varepsilon} u)\right. & +J_{4-n}(2 m \sqrt{\varepsilon} u) \\
& \left.+\frac{1}{4-n}\left[J_{2-n}(2 m \sqrt{\varepsilon} u)-J_{6-n}(2 m \sqrt{\varepsilon} u)\right]\right\} .
\end{aligned}
$$

Hence

$$
\Phi_{1}(\varepsilon)=-\varepsilon \Phi_{0}(\varepsilon)-\frac{m^{2} \varepsilon}{(2 \pi)^{3}} R(\varepsilon)
$$

with

$$
R(\varepsilon) \equiv \lim _{n \rightarrow 4} \int_{1}^{\infty} \frac{d u}{\sqrt{u^{2}-1}} \cdot \frac{J_{2-n}(2 m \sqrt{\varepsilon} u)-J_{6-n}(2 m \sqrt{\varepsilon} u)}{4-n} .
$$

Carrying out the integration over $u$ by means of (A.24), we have

$$
R(\varepsilon)=-\frac{\pi}{4} \lim _{\alpha \rightarrow 0} \frac{1}{\alpha}\left[J_{\alpha-1}(m \sqrt{\varepsilon}) N_{\alpha-1}(m \sqrt{\varepsilon})-J_{\alpha+1}(m \sqrt{\varepsilon}) N_{\alpha+1}(m \sqrt{\varepsilon})\right] .
$$

The limit is calculated by the l'Hôpital rule. Then the use of (A.8) and (A.10) yields

$$
\begin{aligned}
R(\varepsilon)= & \frac{\pi}{4}\left\{J_{1}(m \sqrt{\varepsilon}) \frac{d}{d \alpha}\left[\frac{2 \alpha}{m \sqrt{\varepsilon}} N_{\alpha}(m \sqrt{\varepsilon})\right]_{\alpha=0}\right. \\
& \left.+N_{1}(m \sqrt{\varepsilon}) \frac{d}{d \alpha}\left[\frac{2 \alpha}{m \sqrt{\varepsilon}} J_{\alpha}(m \sqrt{\varepsilon})\right]_{\alpha=0}\right\} .
\end{aligned}
$$

Thus we arrive at (4.15). 
To calculate $\Phi_{j}(\varepsilon)$ for $j \geqq 2$, we apply (B.1) repeatedly. Then

$$
\begin{aligned}
& \int_{0}^{\varepsilon} d v \cdot v^{j} \cdot(\sqrt{v})^{2-n} J_{2-n}(2 m u \sqrt{v}) \\
& =\sum_{k=0}^{j}(-1)^{k} \frac{j !}{(j-k) !} \cdot \frac{\varepsilon^{(2 j+3-n-k) / 2}}{(m u)^{k+1}} J_{3-n+k}(2 m \sqrt{\varepsilon} u) .
\end{aligned}
$$

The right-hand side of (B.9) reduces to

$$
\varepsilon^{j} \sum_{k=0}^{j}(-1)^{k} \frac{j !}{(j-k) !} \cdot \frac{J_{k-1}(2 m \sqrt{\varepsilon} u)}{(m \sqrt{\varepsilon} u)^{k+1}}
$$

for $n=4$.

Now, we note that the following interesting identity holds:

$$
\sum_{k=0}^{j}(-1)^{k} \frac{j !}{(j-k) !} \cdot \frac{J_{k-1}(2 t)}{t^{k}}=\sum_{k=1}^{j-1} c_{j k} J_{2 k+1}(2 t) \text { for } j \geqq 2,
$$

where

$$
c_{j k} \equiv(-1)^{k+1}(2 k+1) \frac{j !(j-2) !}{(j+k) !(j-k-1) !} .
$$

We prove (B.11) by mathematical induction with respect to $j$. For $j=2$, it reduces to

$$
J_{-1}(2 t)-2 t^{-1} J_{0}(2 t)+2 t^{-2} J_{1}(2 t)=J_{3}(2 t),
$$

which is easily verified by means of (A.10) and (A.8). Hence we assume that (B.11) holds. After multiplying its both sides by $t$, we differentiate them with respect to $t$. Then, with the aid of (A.13), the left-hand side becomes

$$
\frac{2 t}{j+1} \sum_{k=1}^{j+1}(-1)^{k} \frac{(j+1) !}{(j-k+1) !} \cdot \frac{J_{k-1}(2 t)}{t^{k-1}} \text {. }
$$

Hence induction completes if

$$
-t J_{1}(2 t)+\frac{j+1}{2 t} \sum_{k=1}^{j-1} c_{j k} \frac{d}{d t}\left[t J_{2 k+1}(2 t)\right]=\sum_{k=1}^{j} c_{j+1, k} t J_{2 k+1}(2 t) .
$$

But it is straightforward (but somewhat tedious) to verify (B.15) by using (A.12) and (A.10). Thus (B.11) has been established.

The use of (B.11) in (B.10) yields

$$
\Phi_{j}(\varepsilon)=-\frac{m^{2}}{(2 \pi)^{3}} \varepsilon^{j} \int_{1}^{\infty} \frac{d u}{\sqrt{u^{2}-1}} \sum_{k=1}^{j-1} c_{j k} \frac{J_{2 k+1}(2 m \sqrt{\varepsilon} u)}{m \sqrt{\varepsilon u}} .
$$

It is now easy to carry out the integration over $u$ by means of (A.10) and (A.24). We then have

$$
\begin{aligned}
\Phi_{j}(\varepsilon)= & -\frac{m^{2}}{(2 \pi)^{3}} \varepsilon^{j}\left(-\frac{\pi}{2}\right) \sum_{k=1}^{j-1} \frac{c_{j k}}{2 k+1}\left[J_{k}(m \sqrt{\varepsilon}) N_{k}(m \sqrt{\varepsilon})\right. \\
& \left.+J_{k+1}(m \sqrt{\varepsilon}) N_{k+1}(m \sqrt{\varepsilon})\right],
\end{aligned}
$$

from which (4.16) follows immediately. 
The calculation of $\Psi_{j}(\varepsilon)$ is quite analogous to that of $\Phi_{j}(\varepsilon)$ though slightly more complicated. From (4.24)-(4.26), $\Psi_{j}(\varepsilon)$ can be written as

$$
\begin{aligned}
\Psi_{j}(\varepsilon)= & \lim _{n \rightarrow 4} \frac{m^{n-2}}{2^{n-2} \pi^{n-1}} \int_{1}^{\infty} \frac{d u}{\sqrt{u^{2}-1}} \int_{0}^{\varepsilon} d v \cdot v^{j}\left[-(\sqrt{v})^{2-n} N_{2-n}(2 m u \sqrt{v})\right. \\
& \left.+(-1)^{j}(2 / \pi)(\sqrt{v})^{2-n} K_{2-n}(2 m u \sqrt{v})\right] .
\end{aligned}
$$

For $j=0$, by means of (A.16) and (A.17), we obtain

$$
\Psi_{0}(\varepsilon)=\frac{m^{2}}{4 \pi^{3}} \int_{1}^{\infty} \frac{d u}{\sqrt{u^{2}-1}} \cdot \frac{N_{1}(2 m \sqrt{\varepsilon} u)-(2 / \pi) K_{1}(2 m \sqrt{\varepsilon} u)}{m \sqrt{\varepsilon} u}
$$

with the aid of (A.8) and (A.9). Note that the additional terms encountered in (A.16) and in (A.17) are cancelled out in (B.19). It is easy to see that (4.27) follows from (B.19) by using (A.10), (A.11), (A.25), and (A.26).

The calculation of $\Psi_{1}(\varepsilon)$ is carried out in parallel with that of $\Phi_{1}(\varepsilon)$. Corresponding to (B.5) with (B.7), we obtain

$$
\Psi_{1}(\varepsilon)=-\varepsilon \tilde{\Psi}_{0}(\varepsilon)+\frac{m^{2} \varepsilon}{2^{4} \pi^{2}} S(\varepsilon)
$$

where $\tilde{\Psi}_{0}(\varepsilon)$ stands for what is obtained from $\Psi_{0}(\varepsilon)$ by changing the signs of its last two terms [this change is owing to $(-1)^{j}$ in (B.18)] and

$$
\begin{aligned}
S(\varepsilon) \equiv & \frac{1}{2} \lim _{\alpha \rightarrow 0} \frac{1}{\alpha}\left\{-\left[J_{\alpha-1}(m \sqrt{\varepsilon})\right]^{2}+\left[N_{\alpha-1}(m \sqrt{\varepsilon})\right]^{2}+\left[J_{\alpha+1}(m \sqrt{\varepsilon})\right]^{2}\right. \\
& \left.-\left[N_{\alpha+1}(m \sqrt{\varepsilon})\right]^{2}-\left[(2 / \pi) K_{\alpha-1}(m \sqrt{\varepsilon})\right]^{2}+\left[(2 / \pi) K_{\alpha+1}(m \sqrt{\varepsilon})\right]^{2}\right\} .
\end{aligned}
$$

It is easy to calculate (B.21) by the l'Hôpital rule together with (A.8)-(A.11). In this way, we can derive (4.28).

Finally, the calculation of $\Psi_{j}(\varepsilon)$ for $j \geqq 2$ is carried out in the same way as that of $\Phi_{j}(\varepsilon)$ for $j \geqq 2$. It is sufficient to note that the same identity as (B.11) holds also for Neumann's Bessel functions and that for the modified Bessel functions it is modified into

$$
\sum_{k=0}^{j} \frac{j !}{(j-k) !} \cdot \frac{K_{k-1}(2 t)}{t^{k}}=\sum_{k=1}^{j-1}(-1)^{k+1} c_{j k} K_{2 k+1}(2 t) \text { for } j \geqq 2 .
$$

\section{Appendix C. Derivation of (4.17)-(4.19) and (4.30)-(4.32)}

Since from (A.5) we have

$$
\begin{aligned}
& \frac{\pi}{2} N_{0}(m \sqrt{\varepsilon}) \sim \log \frac{m \sqrt{\varepsilon}}{2}+\gamma, \\
& \frac{\pi}{2} N_{1}(m \sqrt{\varepsilon}) \sim-\frac{1}{m \sqrt{\varepsilon}}+\frac{m \sqrt{\varepsilon}}{2}\left(\log \frac{m \sqrt{\varepsilon}}{2}+\gamma-\frac{1}{2}\right),
\end{aligned}
$$

it is easy to show (4.17) and (4.18) from (4.14) and (4.15), respectively. 
Since (A.1) and (A.5) yield

$$
(\pi / 2) J_{k}(m \sqrt{\varepsilon}) N_{k}(m \sqrt{\varepsilon}) \sim-1 / 2 k,
$$

(4.16) behaves like

$$
\Phi_{j}(\varepsilon) \sim-\frac{m^{2}}{(2 \pi)^{3}} \varepsilon^{j}\left[\sum_{k=1}^{j} \frac{(-1)^{k} j !(j-2) !}{(j+k) !(j-k) !}+\frac{1}{2(j-1)}\right] \text { for } j \geqq 2 .
$$

Since the well-known formula

$$
{ }_{2} F_{1}(a, b ; c ; 1)=\frac{\Gamma(c) \Gamma(c-a-b)}{\Gamma(c-a) \Gamma(c-b)}
$$

gives us

$$
\sum_{k=1}^{j} \frac{(-1)^{k}(j !)^{2}}{(j+k) !(j-k) !}={ }_{2} F_{1}(-j, 1 ; j+1 ; 1)-1=-\frac{1}{2},
$$

(C.4) reduces to (4.19).

Now, we consider $\Psi_{j}(\varepsilon)$. From (A.6) we see

$$
\begin{aligned}
& K_{0}(m \sqrt{\varepsilon}) \sim-\log \frac{m \sqrt{\varepsilon}}{2}-\gamma, \\
& K_{1}(m \sqrt{\varepsilon}) \sim \frac{1}{m \sqrt{\varepsilon}}+\frac{m \sqrt{\varepsilon}}{2}\left(\log \frac{m \sqrt{\varepsilon}}{2}+\gamma-\frac{1}{2}\right) .
\end{aligned}
$$

By using (C.1), (C.2), (C.7), and (C.8), it is straightforward to show (4.30) and (4.31) for $j=1$ from (4.27) and (4.28), respectively.

It is a rather intricate task to find the small- $\varepsilon$ behavior of $\Psi_{j}(\varepsilon)$ for $j \geqq 2$. From (A.5) and (A.6), neglecting positive-power terms, for $k \geqq 1$ we have

$$
\begin{aligned}
{\left[\frac{\pi}{2} N_{k}(z)\right]^{2} \sim } & \frac{1}{4}\left(\frac{z}{2}\right)^{-2 k}\left[\sum_{l=0}^{k-1} \frac{(k-l-1) !}{l !}\left(\frac{z}{2}\right)^{2 l}\right]^{2}-\frac{1}{k}\left(\log \frac{z}{2}+\gamma\right)+\frac{1}{2 k} \sum_{m=1}^{k} \frac{1}{m}, \\
{\left[K_{k}(z)\right]^{2} \sim } & \frac{1}{4}\left(\frac{z}{2}\right)^{-2 k}\left[\sum_{l=0}^{k-1}(-1)^{l} \frac{(k-l-1) !}{l !}\left(\frac{z}{2}\right)^{2 l}\right]^{2} \\
& -\frac{(-1)^{k}}{k}\left(\log \frac{z}{2}+\gamma\right)+\frac{(-1)^{k}}{2 k} \sum_{m=1}^{k} \frac{1}{m} .
\end{aligned}
$$

Hence the coefficient of $\log (m \sqrt{\varepsilon} / 2)+\gamma$ in $\Psi_{j}(\varepsilon)$ is

$$
-\frac{m^{2}}{\pi^{4}} \varepsilon^{j} \cdot \frac{1-(-1)^{j}}{2}\left[\sum_{k=1}^{j} \frac{(-1)^{k} j !(j-2) !}{(j+k) !(j-k) !}+\frac{1}{2(j-1)}\right] .
$$

As we have seen in (C.4), the quantity in the square bracket of (C.11) equals $1 / 2 j$.

Next, we have to simplify the first term of (C.9). Since positive-power terms are omitted, we have

$$
\left(\frac{z}{2}\right)^{-2 k}\left[\sum_{l=0}^{k-1} \frac{(k-l-1) !}{l !}\left(\frac{z}{2}\right)^{2 l}\right]^{2} \sim \sum_{r=0}^{k-1} a_{k r}\left(\frac{z}{2}\right)^{2 r-2 k}+\sum_{l=1}^{k-1} \frac{1}{l(k-l)},
$$


where

$$
a_{k r} \equiv \sum_{l=0}^{r} \frac{(k-l-1) !(k-r+l-1) !}{l !(r-l) !} .
$$

Let $v$ be a complex number and consider an identity

$$
\sum_{l=0}^{r} \frac{\Gamma(v-l+1) \Gamma(v-r+l+1)}{l !(r-l) !}=\frac{\Gamma(v+1) \Gamma(v-r+1)}{r !}{ }_{2} F_{1}(v-r+1,-r ;-v ; 1) .
$$

Its right-hand side is calculated by means of (C.5). Then taking the limit of $v \rightarrow k-1 \geqq r$, we obtain

$$
a_{k r}=\frac{[(k-r-1) !]^{2}(2 k-r-1) !}{r !(2 k-2 r-1) !} .
$$

On substituting (C.12) with (C.15) in (C.9), we find that the negative-power part in $\left[(\pi / 2) N_{k}(z)\right]^{2}$ is

$$
\frac{1}{4} \sum_{p=1}^{k} \frac{[(p-1) !]^{2}(k+p-1) !}{(k-p) !(2 p-1) !}\left(\frac{z}{2}\right)^{-2 p}
$$

and that the constant term in $\left[(\pi / 2) N_{k}(z)\right]^{2}$ is

$$
\frac{1}{2 k}\left[\sum_{m=1}^{k-1} \frac{1}{m}+\sum_{m=1}^{k} \frac{1}{m}\right]
$$

apart from $-\gamma / k$. The corresponding quantities for $\left[K_{k}(z)\right]^{2}$ are obtained by replacing $z$ by $i z$ in (C.16) and multiplying (C.16) and (C.17) by $(-1)^{k}$.

We show below that the negative-power part of

$$
\sum_{k=1}^{j} \frac{2 k}{(j+k) !(j-k) !}\left\{(-1)^{k}\left[\frac{\pi}{2} N_{k}(z)\right]^{2}-(-1)^{j}\left[K_{k}(z)\right]^{2}\right\}
$$

vanishes exactly. From the above results, it is rewritten as

$$
\sum_{p=1}^{j} \frac{1-(-1)^{j+p}}{2} \cdot \frac{[(p-1) !]^{2}}{(2 p-1) !} b_{j p}\left(\frac{z}{2}\right)^{-2 p}
$$

where

$$
b_{j p} \equiv \sum_{k=p}^{j}(-1)^{k} \frac{k \cdot(k+p-1) !}{(j+k) !(j-k) !(k-p) !} .
$$

Since the $p=j$ term of (C.19) evidently vanishes, it is sufficient to prove

$$
b_{j p}=0 \text { for } p=1,2, \ldots, j-1 .
$$

To show (C.21), we rewrite (C.20) as

$$
\begin{aligned}
b_{j p} & =(-1)^{p}\left[\sum_{l=0}^{j-p} \frac{(-1)^{l}(2 p+l) !}{l !(j+p+l) !(j-p-l) !}-p \sum_{l=0}^{j-p} \frac{(-1)^{l}(2 p+l-1) !}{l !(j+p+l) !(j-p-l) !}\right] \\
& =\frac{(-1)^{p}(2 p) !}{(j+p) !(j-p) !}\left[{ }_{2} F_{1}(2 p+1, p-j ; j+p+1 ; 1)-\frac{1}{2}{ }_{2} F_{1}(2 p, p-j ; j+p+1 ; 1)\right] .
\end{aligned}
$$

From (C.22), we obtain (C.21) by means of (C.5). 

from

Thus the only surviving negative-power part of $\varepsilon^{-j} \Psi_{j}(\varepsilon)$ is the contribution

$$
\left[N_{1}(m \sqrt{\varepsilon})\right]^{2}+(-1)^{j}\left[(2 / \pi) K_{1}(m \sqrt{\varepsilon})\right]^{2} \sim(2 / \pi)^{2}\left[1+(-1)^{j}\right]\left(m^{2} \varepsilon\right)^{-1} .
$$

Finally, we consider the constant term of $\varepsilon^{-j} \Psi_{j}(\varepsilon)$. As is seen from (4.29) and (C.17), we have to calculate

$$
\begin{aligned}
A_{j} & \equiv \sum_{k=1}^{j} \frac{(-1)^{k} j !(j-2) !}{(j+k) !(j-k) !}\left[\sum_{m=1}^{k-1} \frac{1}{m}+\sum_{m=1}^{k} \frac{1}{m}\right]+\frac{1}{2(j-1)} \\
& =\frac{1}{j(j-1)}\left(2 B_{j}-C_{j}\right)+\frac{1}{2(j-1)},
\end{aligned}
$$

where

$$
\begin{aligned}
& B_{j} \equiv \sum_{k=1}^{j} \frac{(-1)^{k}(j !)^{2}}{(j+k) !(j-k) !} \sum_{m=1}^{k} \frac{1}{m}, \\
& C_{j} \equiv \sum_{k=1}^{j} \frac{(-1)^{k}(j !)^{2}}{(j+k) !(j-k) !} \cdot \frac{1}{k} .
\end{aligned}
$$

Since we can write

$$
B_{j}=\left.(\partial / \partial b)_{2} F_{1}(-j, b ; j+1 ; 1)\right|_{b=1},
$$

(C.5) yields

$$
B_{j}=-\frac{1}{2}\left(\frac{1}{j}+\frac{1}{j+1}+\ldots+\frac{1}{2 j-1}\right)
$$

On the other hand, we have

$$
\begin{aligned}
C_{j} & =\int_{0}^{1} d x \cdot x^{-1}\left[{ }_{2} F_{1}(-j, 1 ; j+1 ; x)-1\right] \\
& =\int_{0}^{1} d x \cdot x^{-1}\left[j \int_{0}^{1} d t(1-t)^{j-1}(1-t x)^{j}-1\right] \\
& =j \int_{0}^{1} d t(1-t)^{j-1} \int_{0}^{1} d x \cdot x^{-1}\left[(1-t x)^{j}-1\right] \\
& =j \int_{0}^{1} d t(1-t)^{j-1} \int_{0}^{t} d y \cdot y^{-1}\left[(1-y)^{j}-1\right] \\
& =\int_{0}^{1} d t(1-t)^{j} \cdot t^{-1}\left[(1-t)^{j}-1\right] \\
& =-\left(\frac{1}{j+1}+\frac{1}{j+2}+\ldots+\frac{1}{2 j}\right) .
\end{aligned}
$$

Substitution of (C.28) and (C.29) in (C.24) yields

$$
A_{j}=(j+1) / 2 j^{2} \text {. }
$$


Collecting the results (C.11), (C.23), and (C.30), we obtain

$$
\begin{aligned}
\Psi_{j}(\varepsilon) \sim & -\frac{m^{2} \varepsilon^{j}}{\pi^{4}} \cdot \frac{1-(-1)^{j}}{2} \cdot \frac{1}{2 j}\left(\log \frac{m \sqrt{\varepsilon}}{2}+\gamma\right) \\
& +\frac{m^{2} \varepsilon^{j}}{2^{4} \pi^{2}} \cdot\left(\frac{2}{\pi}\right)^{2}\left[\frac{1+(-1)^{j}}{2} \cdot \frac{2 \varepsilon^{-1}}{(j-1) m^{2}}+\frac{1-(-1)^{j}}{2} \cdot \frac{j+1}{j^{2}}\right] \\
& \text { for } j \geqq 2 .
\end{aligned}
$$

\section{References}

1. 't Hooft, G., Veltman, M.: Nucl. Phys. B 44, 189-213 (1972); Butera,P., Cicuta, G.M., Montaldi,E.: Nuovo Cimento 19A, 513-528 (1974). Further references are contained in the latter

2. Schwartz,L.: Théorie des Distributions, Paris: Herman \& Cie. 1950

3. Nakanishi, N.: Progr. theoret. Phys. Suppl. 51, 1-95 (1972)

4. Feinberg, G.: Phys. Rev. 159, 1089-1105 (1967)

5. Frishman, Y.: Phys. Reports 13, 1-52 (1974)

6. Nakanishi, N.: J. math. Phys. 5, 1458-1473 (1964)

7. Wightman, A. S.: Unpublished; Tarski, J.: J. math. Phys. 5, 1713-1722 (1964)

8. Sato, M.: J. Faculty of Science, Univ. of Tokyo 8, 139-193 (1959); 8, 387-437 (1960); Pham, F. (ed.): Hyperfunctions and Theoretical Physics, Berlin-Heidelberg-New York: Springer 1975

9. Erdélyi, A.: Higher Transcendental Functions, Vol. 2, New York-Toronto-London: McGrawHill 1953

10. Erdélyi, A.: Tables of Integral Transforms, Vols. 1 and 2, New York-Toronto-London: McGrawHill 1954

Communicated by R. Haag

Received November 30, 1975 